\title{
Identification of genes related to high royal jelly production in the honey bee (Apis mellifera) using microarray analysis
}

\author{
Hongyi Nie ${ }^{\# 1}$, Xiaoyan $\mathrm{Liu}^{\# 1}$, Jiao Pan ${ }^{\# 2}$, Wenfeng $\mathrm{Li}^{2}$, Zhiguo $\mathrm{Li}^{1}$, Shaowu Zhang ${ }^{1,3}$, Shenglu Chen ${ }^{2}$, \\ Xiaoqing Miao ${ }^{1}$, Nenggan Zheng ${ }^{2,4}$ and Songkun $\mathrm{Su}^{1}$ \\ ${ }^{1}$ College of Bee Science, Fujian Agriculture and Forestry University, Fuzhou, China. \\ ${ }^{2}$ College of Animal Sciences, Zhejiang University, Hangzhou, China. \\ ${ }^{3}$ Research School of Biology, College of Medicine, Biology and Environment, The Australian National \\ University, Canberra, Australia. \\ ${ }^{4}$ Qiushi Academy for Advanced Studies, Zhejiang University, Hangzhou, China.
}

\begin{abstract}
China is the largest royal jelly producer and exporter in the world, and high royal jelly-yielding strains have been bred in the country for approximately three decades. However, information on the molecular mechanism underlying high royal jelly production is scarce. Here, a cDNA microarray was used to screen and identify differentially expressed genes (DEGs) to obtain an overview on the changes in gene expression levels between high and low royal jelly producing bees. We developed a honey bee gene chip that covered 11,689 genes, and this chip was hybridised with cDNA generated from RNA isolated from heads of nursing bees. A total of 369 DEGs were identified between high and low royal jelly producing bees. Amongst these DEGs, 201 (54.47\%) genes were up-regulated, whereas 168 $(45.53 \%)$ were down-regulated in high royal jelly-yielding bees. Gene ontology (GO) analyses showed that they are mainly involved in four key biological processes, and pathway analyses revealed that they belong to a total of 46 biological pathways. These results provide a genetic basis for further studies on the molecular mechanisms involved in high royal jelly production.
\end{abstract}

Keywords: honeybee, royal jelly production, gene chip, molecular marker, differentially expressed genes.

Received: February 02, 2017; Accepted: May 04, 2017.

\section{Introduction}

The honey bee (Apis mellifera) is an important insect that generates high economic and ecological values for humans as a key pollinator of crops (Morse and Calderone, 2000; Klein et al., 2007) and producer of bee products, including honey, royal jelly (RJ), pollen, propolis and beeswax (Schmidt, 1997). A published genome (The Honey Bee Genome Sequencing Consortium, 2006) and its biological characteristics have also contributed to the recent emergence of honey bees as a new model organism for research in diverse areas of learning and memory (Zhang et al., 2006; Adler, 2013), division of labour (Ament et al., 2008, 2010), caste differentiation (Barchuk et al., 2007; Li et al., 2013), genetics (Page Jr et al., 2012) and breeding (Hyink et al., 2013).

Royal jelly is the principal food consumed by larval and adult honey bee queens and is secreted by the hypo-

Send correspondence to Songkun Su. College of Bee Science, Fujian Agriculture and Forestry University, No.15 Shangxiadian Road, Cangshan District, 350002 Fuzhou, P. R. China. E-mail: susongkun@zju.edu.cn.

"These authors contributed equally to this work. pharyngeal and mandibular glands of nursing worker bees. Royal jelly is rich in various nutrients beneficial to humans, including proteins, sugars, vitamins and a large number of bioactive substances, such as 10-hydroxy-2-decenoic acid (Viuda-Martos et al., 2008). Royal jelly is used as a health food and natural cosmetic in many countries (Ramadan and Al-Ghamdi, 2012). The market value of royal jelly is considerably higher than that of honey or pollen (Bogdanov, 2011; Ramadan and Al-Ghamdi, 2012), and royal jelly production has become a major income source of many beekeepers in China.

China is the largest royal jelly producer and exporter in the world, with current exports accounting for more than $90 \%$ of the total international trade of royal jelly, and this value resulted from the development, establishment and refinement of a high royal jelly-producing strain of the honey bee (Apis mellifera ligustica) (Cao et al., 2016). Zhenongda No. 1 is a honey bee strain with higher production of honey and royal jelly than wild-type Apis mellifera ligustica (Chen et al., 2002). This strain is well known for its genetic differences and royal jelly production ability. Previous studies have identified the genetic markers of high royal 
jelly-producing bees, including morphological $(\mathrm{Su}$ and Chen, 2003), cytological markers (Chen et al., 2005) and biochemical markers (Zhang et al., 2007; Li et al., 2008; Jianke et al., 2010; Zheng et al., 2010).

However, the molecular mechanisms underlying genetic differences in the ability of honey bees to produce royal jelly remain unknown. Microarray chips are a high throughput and mass data processing technology used to analyse differentially expressed genes (DEGs) in specific biological processes. Microarrays have been extensively used in the study of honey bees, including age-related division of labour (Kucharski and Maleszka, 2002; Grozinger et al., 2003; Whitfield et al., 2003), caste differentiation (Barchuk et al., 2007), susceptibility to Varroa parasitism (Navajas et al., 2008), as well as immunity and disease (Evans, 2006). Dozens of crucial genes can be screened using DNA microarrays for further functional identification. In the present study, 369 DEGs were identified contrasting high royal jelly producing bees and low royal jelly producing bees using chip analysis. This study is the first to conduct a large-scale analysis on gene expression differences between high royal jelly producing bees and low royal jelly producing bees. The results provide a broad perspective on the genes involved in royal jelly production, thereby providing further insights into the mechanism of the royal jelly-producing trait.

\section{Material and Methods}

\section{Honey bee colonies and sample collection}

High royal jelly-producing colonies (Zhenongda No. $1)$, which were derived from an Italian honey bee subspecies (Apis mellifera ligustica), were obtained from the experimental apiary of Zhejiang University, Hangzhou, China. Local Italian bees with a low royal jelly-producing trait were obtained from Miao Siwei Apiary, Simao County, Yunnan Province.

To reduce differences in their genetic background between high and low royal jelly producing colonies, the virgin queens of the low royal jelly-yielding strain were delivered to the experimental apiary of Zhejiang University and there mated naturally with local drones of the high royal jelly-yielding strain to generate F1 progeny. Then, virgin queens were raised from F1 offspring and backcrossed to drones of the high royal jelly-yielding strain. The colonies of high royal jelly producing bees and low royal jelly producing bees were derived from the back-cross progeny colonies, and the royal jelly production of the back-cross progeny colonies was measured prior to sample collection. The collection method of royal jelly was performed as described in a previous report, with minor modifications (Jianke et al., 2010). Each colony was provided with two plastic strips harbouring 128 queen cell cups with grafted one-day-old larvae, and royal jelly was collect after $72 \mathrm{~h}$ of larval grafting. Royal jelly collections were done three times for each colony, and the samples were weighed with a digital scale (Mettler Toledo, Colombus, OH, USA; accurate to $0.001 \mathrm{~g}$ ). Four colonies of high royal jelly producing bees with a high royal jelly production and four colonies of low royal jelly producing bees with a low royal jelly production were selected from the back-cross progeny colonies and then used as material for chip and qRT-PCR analyses. The nurses were caught at the time when they entered the queen cell cups and were feeding the larvae. All of the collected bees were frozen immediately in liquid nitrogen and then stored at $-80^{\circ} \mathrm{C}$ until analysis.

\section{Microarray construction}

A honey bee chip was designed following the Agilent eArray Design guidelines. It contained 11,689 genes from GenBank

(http://www.ncbi.nlm.nih.gov/gene/?term=honey bee) and gene sequences obtained from our lab sequence results, not yet deposited in NCBI. Each gene had a 60 bp sequence included in the oligonucleotide microarray construction done by Advanced Throughput Inc. (Shanghai, China). The details of probes are provided as Supplementary material (Table S1).

\section{RNA extraction, One-Color labelling and microarray hybridisation}

Heads of nursing bees collected from the honey bee samples were cleaned in PBS solution to remove other tissues. Total RNA was extracted from pools of 30 heads per colony using TRIzol (Invitrogen, Waltham, MA, USA) according to the procedures of a previous study (Liu et al., 2011). RNA concentration and quality were assessed using a NanoDrop 2000 spectrophotometer (NanoDrop 2000, Thermo Fisher Scientific, Waltham, MA, USA) and an Agilent 2100 Bioanalyzer, respectively, and were replicated for four colonies each for high royal jelly producing bees and low royal jelly producing bees.

The One-Color Quick Amp Labelling Kit (Agilent, Santa Clara, CA, USA) was used to generate fluorescent cRNA according to the manufacturer's instructions. Aliquots of $200 \mathrm{ng}$ of total RNA from each sample were used in a protocol described by Ferrari et al. (2011), and 1.5 $\mu \mathrm{g}$ of Cy3-labelled cRNAs were obtained from each sample. The cRNAs were fragmented for $15 \mathrm{~min}$ and hybridised for $17 \mathrm{~h}$ at $65^{\circ} \mathrm{C}$ in $2 \mathrm{GE}$ hybridisation buffer HI-RPM following the instruction of Agilent Gene Expression Hybridization Kit (Agilent).

\section{Chip scanning and data analysis}

After hybridisation, the slides were disassembled using GE wash buffer 1 and washed twice with GE wash buffers 1 and 2 for $1 \mathrm{~min}$ at room temperature. The chip was then scanned with an Agilent scanner. Raw data were obtained and data normalisation was conducted using the Agilent Feature Extraction Software (Zahurak et al., 2007). 
Standardized data were provided as Table S2. DEGs with at least twofold changes between high royal jelly producing bees and low royal jelly producing bees were screened for analysis ( $\log _{2}$ ratio $>1$; ratio: the fluorescence intensity of high royal jelly producing bees/low royal jelly producing bees) following methods described previously (Gerhold et al., 2001). Cluster analysis was performed using Cluster 3.0 and TreeView software.

\section{Bioinformatics analysis}

GO and pathway analyses were performed to track the functional annotation and biological pathways of DEGs using ClueGo (Bindea et al., 2009) referencing the DroSpeGe database (Gilbert, 2007) and KEGG.

\section{qRT-PCR assays of the selected genes}

Total RNA extraction and cDNA synthesis were performed as described previously (Nie et al., 2014). The primers, which were designed using Primer Premier 5.0, are listed in Table S3. qRT-PCR in our study was performed using the LineGeneK PCR System (Bioer Technology Co., Ltd., Hangzhou, China). The PCR mix $(20 \mu \mathrm{L}$ total) consisted of $2 \mu \mathrm{L}$ of $250 \mathrm{ng} / \mu \mathrm{L}$ cDNA template, 10 $\mu \mathrm{L}$ THUNDERBIRD SYBR qPCR Mix (QPS-201, TOYBO, Osaka, Japan), $1 \mu \mathrm{L}$ of $10 \mu \mathrm{M}$ forward primer, 1 $\mu \mathrm{L}$ of $10 \mu \mathrm{M}$ reverse primer and $6 \mu \mathrm{L}$ of nuclease-free water. The reactions were run as follows: $95^{\circ} \mathrm{C}$ for $1 \mathrm{~min}, 40$ cycles of $15 \mathrm{~s}$ at $95^{\circ} \mathrm{C}$ and $45 \mathrm{~s}$ at $60^{\circ} \mathrm{C}$. GAPDH (forward: GATGCACCCATGTTTGTTTG; reverse: TTTGCAGAAGGTGCATCAAC) was used as an internal control gene because of its stable expression level in the heads of honey bees (Scharlaken et al., 2008). All reactions were run in triplicates. The $\mathrm{Ct}$ values were analysed using default threshold settings, and the mean $\mathrm{Ct}$ values of each triplicate sample were used for the subsequent analysis. The relative quantification of the selected gene expression was calculated using $2^{-\Delta \Delta \mathrm{Ct}}$ method (Livak and Schmittgen, 2001).

\section{Statistical analysis}

Data analysis of the royal jelly yield between high royal jelly producing bees and low royal jelly producing bees used for gene chip and qRT-PCR was performed using the Data Processing System (DPS) software (Tang and Zhang, 2013). Independent-sample $t$ tests implemented in SPSS 13.0 software were performed to analyse the results.

\section{Results}

\section{DEGs between high royal jelly producing bees and} low royal jelly producing bees

Four colonies of high royal jelly producing bees and four colonies of low royal jelly producing bees were selected from the back-cross progeny colonies. Royal jelly production of high royal jelly producing bees was $122 \mathrm{~g}$ to $177 \mathrm{~g}$ with an average of $145.75 \mathrm{~g}$. The royal jelly production of low royal jelly producing bees was $33 \mathrm{~g}$ to $93 \mathrm{~g}$ with an average of $66.75 \mathrm{~g}$. Production of royal jelly between high royal jelly producing bees and low royal jelly producing bees exhibited a significant difference (Figure 1). This finding indicated that the colonies were reliable for the subsequent study. After hybridisation, scanning and data analysis, 369 DEGs between high royal jelly producing bees and low royal jelly producing bees with at least twofold changes were identified (Table S4). Amongst these modulated genes, 201 (54.47\%) were up-regulated, whereas 168 $(45.53 \%)$ were down-regulated in high royal jelly producing bees. Hierarchical clustering analysis showed that four high royal jelly bee colonies were clustered, and the other four low royal jelly bee colonies were clustered, indicating that the samples used for microarray are reliable (Figure 2).

\section{Gene ontology (GO) and Kyoto Encyclopaedia of Genes and Genomes (KEGG) pathway analyses}

$\mathrm{GO}$ analysis was used to classify possible functions of genes. Based on sequence homology, these functions were broadly categorised into four groups according to biological process, namely, developmental regulation of organic tissue, synthesis and deposition of nutrients, oxidoreduction coenzyme and glucose metabolism, and biosynthesis and metabolism of organic acid (Figure 3A). According to molecular functions, these were divided into two categories, namely, neuropeptide receptor activity and metabolic enzyme activity. The two categories were further broken down into 12 detailed groups: neuropeptide binding, peptide receptor activity, $\mathrm{G}$ protein-coupled receptor activity (GPCR), neuropeptide receptor activity, RNA heli-

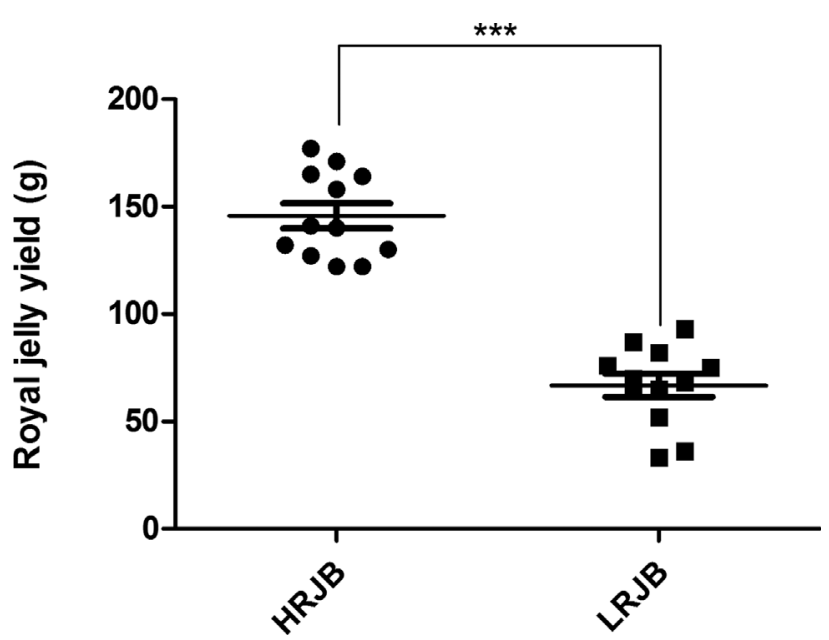

Figure 1 - Royal jelly yield of high royal jelly producing bees (HRJB) and low royal jelly producing bees (LRJB). Four colonies of high royal jelly producing bees and four colonies of low royal jelly producing bees were selected from the back-cross progeny colonies. The royal jelly collections were performed three times for each colony and weighed with a digital scale. Independent-sample $t$-tests were performed to analyse the results using the SPSS 13.0 software. $\left.{ }^{*} \mathrm{p}<0.001\right)$ 


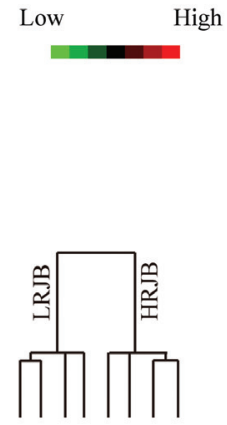

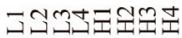

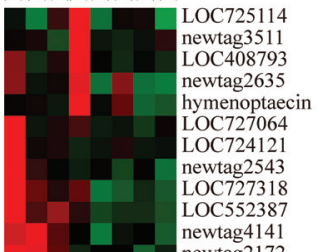

newtag4141
newtag2172

LOC724959

LOC410861

LOC727151

LOC726782

newtag 5787

newtag5088

LOC724572

LOC551746
LOC724495

newtag5367

newtag1293

LOC551672
LOC724851
LOC408515
newtag3362
LOC727349

newtag3362
LOC727349
newtag2152
LOC410373

LOC410373
LOC411160
LOC726019
newtag4299

newtag4299
newtag 2572
LOC 25812

LOC725812

LOC408838

LOC408838
LOC727557
LOC412338
LOC552565
LOC 726542

LOC552565
LOC726542
LOC725716
LOC410882
LOC727598
LOC726935

C726935

lk-1

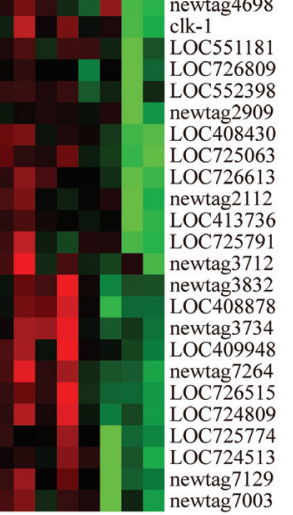

LOC725157
LOC726044 LOC 409868
LOC727374 LOC727374
newtag1336 LOC724593 LOC410796 LOC725085 newtag2060 ewtag2947 LOC552683
newtag6982 OC727482 LOC552142 LOC552142 newtag5043 LC412624 OC725840 LOC725880 LOC551544 LOC724650 LOC 411525 LOC 411525
LOC727257 LOC727257 LOC726751 (16-113 LOC411852 newtag7589 LOC413296 OC4 40896 OC4 13908 OBP11

newtag 4796 LOC 551327 LOC 552418 LOC 412487
LOC552228 LOC552228 LOC 413964
LOC 408807 newtag 1238 newtag1238 OC408734 LOC727640 LOC724656 LOC724656 LOC408691 LOC 410753
LOC725113 LOC 725113 newtag2496 newtag3735
LOC413577
LOC725198 LOC725198 OBP-8

LOC726008

LOC726008
LOC726023 LOC726023 LOC725863
LOC725010 LOC725010
LOC551908 LOC551908 LOC725007 newtag3535 LOC 551180 LOC551180
LOC551599 LOC 551715 LOC551715 LOC 410820 LOC 413408 LOC 413408
LOC 409148 LOC 551670 LOC727058 LOC727058 newtag 2562 newtag2562 LOC724351 LOC 413618 LOC 551973 LOC410308 LOC550794 LOC724405 LOC551961 LOC551785 LOC726768 LOC408331 LOC552795 LOC 410132 LOC 412166 LOC 413727 newtag 1140 LOC 412636 LOC 412082 LOC 413457 LOC 408555 LOC408749 LW Rh LOC 410155 LOC 408790 LOC 411361

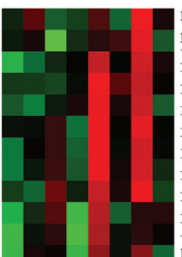

ewtag6813 wag6813 LOC726155 LOC72669 LOC412573 LOC551323 LOC551133 LOC552685 LOC727095 LOC409252 newtag6584

LOC409296 LOC726096 LOC412188 LOC 408783 LOC 411348 LOC411348 LOC 72887 LOC413693 LOC552677 LOC408539 LOC725943 LOC552532 newtag2963 LOC726229 newtag7293 LOC 5513 LOC40834 LOC412112 LOC411320 LOC552686 LOC41076 newtag 4952 LOC72416 LOC409313 LOC727021 LOC41068 newtag 3663 LOC725511 LOC724946 LOC724946
LOC724817 LOC724817
LOC 409090 LOC725048
LOC726402 LOC725169 LOC726929 LOC412520 LOC4 12520
LOC5 11755 LOC552586 LOC409295 LOC727082 RJP57-2 LOC724116 LOC726992 LOC725443 LOC411766 newtag6678 newtag6678 LOC 414038 LOC726901 LOC412172 newtag 2397 newtag 4280 LOC725865 newtag 1634 LOC727397 LOC413196 newtag3258 LOC72449 Hsp90 LOC725019 LOC413775 newtag4627 newtag3389
LOC 408285 LOC726496 csd LOC409103 newtag 4037 LOC72767 LOC726203 newtag2563 newtag6435 LOC551462 newtag 1543 LOC55095 OBP14 LOC727144 LOC409898 creb gene LOC41216 LOC41294 LOC727027 LOC72433

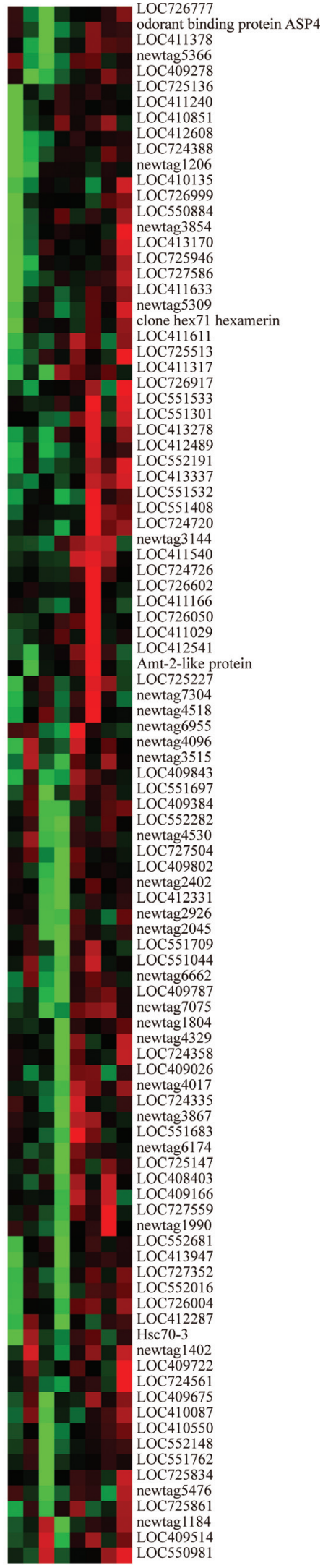

Figure 2 - Hierachical clustering analysis of 369 differently expressed genes. Each horizontal row indicates a gene, and each vertical column represents a colony. In the top of the figure, the tree diagram represents the eight colony samples, which are divided into two groups: high royal jelly producing bees (HRJB) and low royal jelly producing bees (LRJB). Red represents up-regulated genes in HRJB; green represents down-regulated genes in HRJB. Light shades reflect different levels of up- or down-regulated genes. 
case activity, peroxidase activity, scavenger receptor activity, sugar binding, $\mathrm{N}$-acetyl transferase activity, $\mathrm{N}$-acyl transferase activity, oxidoreductase activity, ligase activity (Figure 3B).

We mapped DEGs to the reference canonical pathways in KEGG to identify the biological pathways that par- ticipate in royal jelly production. They were involved in 46 KEGG metabolic pathways, including 4 signalling pathways (i.e., Wnt signalling pathway, hedgehog signalling pathway, TGF-beta signalling pathway and neuroactive ligand-receptor interaction pathway), 35 nutrient anabolic

A

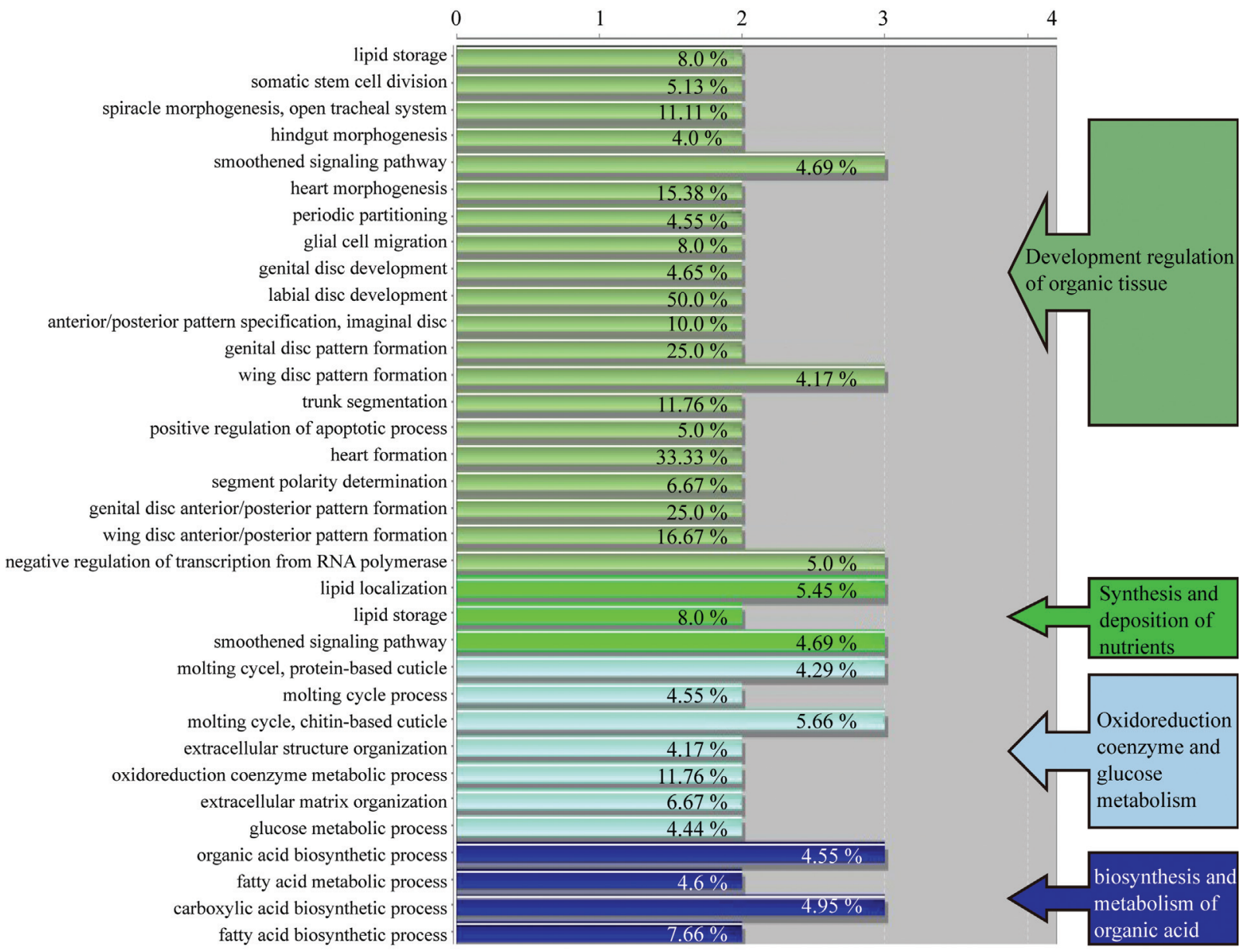

B

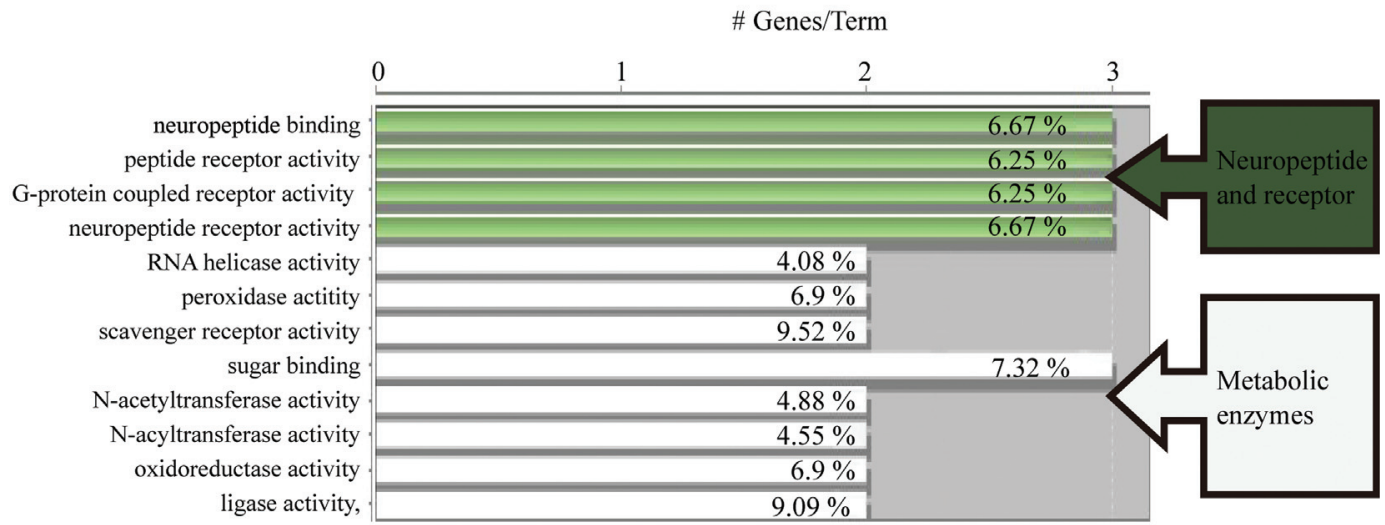

Figure 3 - Analysis on functional enrichment of the differently expressed genes in the high royal jelly producing bees (HRJB) and low royal jelly producing bees (LRJB). Gene ontology (GO) pathway terms specific for differently expressed genes. The bars represent the number of genes associated with the terms. The percentage of genes per term is shown as a bar label. (A) Biological process enrichments of the identified genes. The gene functions are listed on the left and the biological process in which these genes are involved is shown on the right. (B) Molecular function enrichments of the identified genes. 
pathways and seven protein processes and modifications (Table S5).

\section{Validation with quantitative real-time polymerase chain reaction (qRT-PCR)}

Ten genes were randomly selected for confirmation using qRT-PCR to verify the accuracy of the identification of the DEG in the chip data. The 10 genes were significantly up-regulated in high royal jelly producing bees via qRT-PCR analysis (Figure 4), which was consistent with chip data expression profiling analysis. This observation indicated the reliability of our chip expression profiling analysis. In NCBI, these 10 genes were annotated as follows: dopamine receptor type D2 (Dop2) (GI: 20336614); Amt-2-like protein (GI: 67043607); similar to CG8862-PA (LOC551715) (GI: 110755554); clone hex71 hexamerin (hex71) (GI: 149939402); hypothetical protein LOC726515 (LOC726515) (GI: 110759535); similar to lethal (1) G0168 CG33206-PA, isoform A (LOC411348) (GI: 110750767); similar to SHC-adaptor protein CG3715-PA (LOC412172) (GI: 66520065); similar to CG1998-PA, transcript variant 1 (LOC409360) (GI: 110749006); similar to LDLa domain containing chitin binding protein 1 CG8756-PD, isoform $\mathrm{D}$, transcript variant 1 (LOC551323) (GI: 110760992) and similar to multidrug resistance-associated protein 5 (LOC413947) (GI: 66538119). As they were significantly up-regulated in high royal jelly producing bees, these genes could play an important role in royal jelly production of Apis mellifera.

\section{Discussion}

In this study, 369 DEGs were identified between high royal jelly producing bees and low royal jelly producing bees using chip analysis. The KEGG pathway of 201 up- regulated genes in the high royal jelly producing bees were involved in protein synthesis (ribosome, proteasome, aminoacyl-tRNA biosynthesis), amino acid metabolism (glycine, serine and threonine metabolism, arginine and proline metabolism, cysteine and methionine metabolism), nucleotide and fatty acid metabolism (purine metabolism, pyrimidine metabolism, fatty acid metabolism), sugar metabolism (galactose metabolism, amino sugar and nucleotide sugar metabolism), signal transduction (hedgehog signaling pathway, phosphatidylinositol signaling system), oxidation (oxidative phosphorylation, peroxisome), transporter ( $\mathrm{ABC}$ transporters). This suggests that repeated selection pressure for high royal jelly production has affected different pathways.

Compared to the proteome data on hypopharyngeal gland development comparing Italian and royal jelly producing worker honeybees (Jianke et al., 2010), we identified the same three genes: major royal jelly protein 4,60 $\mathrm{kDa}$ heat shock protein and heat shock $70 \mathrm{kDa}$ protein cognate 3; and some similar genes, including ribosomal protein (similar to ribosomal protein L5, ribosomal protein L40, 40S ribosomal protein S29-like), skeleton (actin-related protein 2), and proteasome (proteasome $25 \mathrm{kDa}$ subunit) in this microarray. Major royal jelly protein 4 is a major protein for total royal jelly (Schmitzová et al., 1998; Albert et al., 1999), and it was found up-regulated in high royal jelly bees at the transcriptional and proteomic levels, indicating that the content of major royal jelly protein might be increased. Furthermore, the data for most of the ribosomal proteins, heat shock proteins and proteasome found increased in high royal jelly bees, were also consistent with previous studies (Mao et al., 2009; Jianke et al., 2010; Ji et al., 2014), suggesting that these genes might accelerate protein biosynthesis during nursing behaviour. Interestingly,

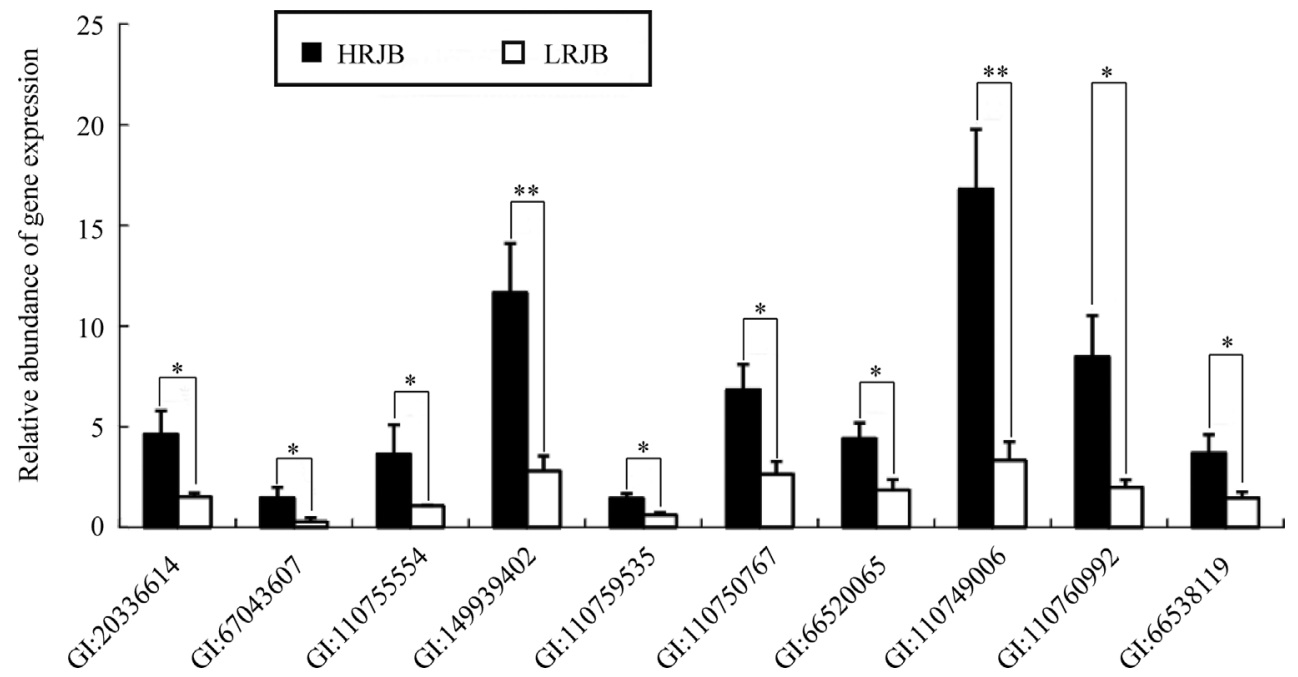

Figure 4 - Validation of the differential expression of ten genes in high royal jelly producing bees (HRJB) and low royal jelly producing bees (LRJB) by qRT-PCR analysis. Four colonies of HRJB with a high royal jelly production and four colonies of LRJB with a low royal jelly production were selected from the back-cross progeny colonies, of which heads of nursing bees $(n=30)$ were collected per colony were used as materials for qRT-PCR analyses. Independent-sample $t$-tests were performed to analyse the results using the SPSS 13.0 software. ${ }^{*} p<0.05 ; * * p<0.01$ ) 
odorant binding protein 4 (OBP4), OBP14 and odorant receptor 22 were up-regulated expressed in the high royal jelly bees, indicating that these genes may easily perceive some chemical signals to make worker manifest nursing behaviours.

Storage protein hex 71 , also called hex $70 a$, belongs to the hexamerin family. Hexamerins are synthesised in fat body cells and are secreted into the hemolymph where they accumulate (Telfer and Kunkel, 1991). hex 71 is involved in nutrient uptake and storage (Braun and Wyatt, 1996). hex71 expression is increased after adult emergence, maintains a high level of transcripts in adult workers (1 to 15 days), and is decreased when nurse bees become forage bees (18 to 28 days old) (Martins et al., 2008). This pattern approximately coincides with the timing of the nursing behaviour. This observation indicated that hex71 may be closely related to the feeding behaviour of adult worker bees. However, Hex71 protein cannot be used as an amino acid resource for hypopharyngeal gland activity and royal jelly production because this gene is not expressed in the hypopharyngeal gland tissue of honey bees (Martins et al., 2008). Previous studies showed that nutrient metabolism and storage conditions may have a strong effect on the transformation of nurse bees into forager bees (Toth et al., 2005). hex71 expression may be closely related to fat body metabolic activity and may play an important role in the physiological development of adult worker bees (Martins et al., 2010). Therefore, we hypothesise that the hex 71 gene is indirectly involved in the synthesis and secretion of royal jelly, but increases the royal jelly yield of a colony by postponing the transition of nurse bees to foraging bees.

Dopamine is a neurotransmitter involved in the regulation of various physiological processes of the central nervous system of many organisms (Schultz, 1992). Dopamine can regulate the motion, circadian rhythm, growth and development, sexual behaviour, endocrine system and cognitive behaviour of insects (Wise, 2004). In honey bees, dopamine production is closely correlated with learning and memory, as well as with movement (Vergoz et al., 2007; Nomura et al., 2009). Dopamine works through membrane receptors (dopamine receptors). In our study, dop 2 was found up-regulated in the high royal jelly producing bees. Previous studies indicated that dopamine regulates the development of honey bee antennal neurons (Perk and Mercer, 2006). We hypothesise that dop 2 is associated with dopamine promoting the development of honey bee antennal neurons. These neurons enable worker bees to find and feed larvae fast and efficiently in the queen cells, thereby increasing the feeding rate and quantity of royal jelly deposited in queen cells, and hence, could ultimately affect the royal jelly yield of the entire colony.

Amt-2-like protein belongs to members of the conserved ammonium transporter (Amt) family. Ammonia transport across biological membranes is a critical feature of nitrogen metabolism, and Amt plays an important role in olfactory signalling (Menuz et al., 2014). In the present study, Amt-2-like protein (GI: 67043607) was up-regulated in the high royal jelly producing bees. Similarly, an aminomethyltransferase (GI: 66523499) gene that participates in the nitrogen metabolism pathway, was also up-regulated in the high royal jelly producing bees. We hypothesise that Amt-2-like may affect olfactory signalling via ammonia transport. Our data indicate that Amt-2-like protein has a critical role in royal jelly production.

In addition to the aforementioned three genes, heat shock protein 90 (HSP90, GI: 229892247) and HSP60 (GI: 110763844) were also up-regulated in the high royal jelly producing bees. HSPs function as binding proteins and molecular chaperones and assist in the folding and processing of new proteins. Several heat shock proteins are expressed in the early development of hypopharyngeal glands in adult worker honey bees, and these proteins reach their peak expression levels between the age of 6 and 12 days (Feng et al., 2009). Hypopharyngeal glands, which contain secretory cells in large quantities, are the main organs involved in the secretion of royal jelly (Ohashi et al., 1997). The key secretion period occurs when adult honey bees are between 6 and 12 days old. Accordingly, HSP90 and HSP60 may protect the royal jelly protein secretion activity of the hypopharyngeal gland by assisting in the correct folding of proteins, thereby contributing to a high royal jelly production.

\section{Conclusion}

In this study, we measured the production of royal jelly in high royal jelly producing bees and low royal jelly producing bee, putting in evidence a significant difference between the two groups. A total of 369 DEGs were identified between high royal jelly producing bees and low royal jelly producing bees using a cDNA microarray. These DEGs are involved in 46 pathways. This is first comprehensive transcriptome database revealing genes that are differentially expressed between high royal jelly bees and low royal jelly bees. Our results provide new insights into the molecular mechanism of royal jelly secretion and also serve as an extensive novel resource for screening molecular markers to accelerate molecular breeding of high royal jelly bees.

\section{Acknowledgments}

This work was supported by National Natural Science Foundation of China (No.30571409), Educational and scientific research program for young and middle-aged instructor of Fujian province (No.JAT160161) and the earmarked fund for Modern Agro-industry Technology Research System (No.CARS-45-KXJ3). The microarray and bio-information analysis work were performed by Shanghai Advanced Throughput Inc. of China. We thank Dr. Zachary Y. Huang and Dr. Weifeng Huang for their helpful suggestions and improvement on the manuscript. We thank 
Shuangjin Dai, Yunjuan Hu, Qin Chen, Li Li and Limin Yan for their help in research.

\section{References}

Adler EM (2013) Tasting the bitter and the sweet, honeybee memories, and visualizing calcium throughout entire astrocytes. J Gen Physiol 141:511-512.

Albert S, Bhattacharya D, Klaudiny J, Schmitzová J and Simúth J (1999) The family of major royal jelly proteins and its evolution. J Mol Evol 49:290-297.

Ament SA, Corona M, Pollock HS and Robinson GE (2008) Insulin signaling is involved in the regulation of worker division of labor in honey bee colonies. Proc Natl Acad Sci U S A 105:4226-4231.

Ament SA, Wang Y and Robinson GE (2010) Nutritional regulation of division of labor in honey bees: Toward a systems biology perspective. Wiley Interdiscip Rev Syst Biol Med 2:566-576.

Barchuk AR, Cristino AS, Kucharski R, Costa LF, Simões ZL and Maleszka R (2007) Molecular determinants of caste differentiation in the highly eusocial honeybee Apis mellifera. BMC Dev Biol 7:70.

Bindea G, Mlecnik B, Hackl H, Charoentong P, Tosolini M, Kirilovsky A, Fridman W-H, Pagès F, Trajanoski Z and Galon J (2009) ClueGO: A Cytoscape plug-in to decipher functionally grouped gene ontology and pathway annotation networks. Bioinformatics 25:1091-1093.

Bogdanov S (2011) Royal jelly, bee brood: Composition, health, medicine: A review. Bee Product Sci 4:1-30.

Braun RP and Wyatt GR (1996) Sequence of the hexameric juvenile hormone-binding protein from the hemolymph of $\mathrm{Lo}$ custa migratoria. J Biol Chem 271:31756-31762.

Cao LF, Zheng HQ, Pirk CW, Hu FL and Xu ZW (2016) High royal jelly-producing honeybees (Apis mellifera ligustica) (Hymenoptera: Apidae) in China. J Econ Entomol 109:510514.

Chen S, Su S and Lin X (2002) An introduction to high-yielding royal jelly production methods in China. Bee World 83:6977.

Chen SL, Li JK, Zhong BX and Su SK (2005) Microsatellite analysis of royal jelly producing traits of Italian honeybee (Apis mellifera Liguatica). Yi Chuan Xue Bao 32:1037-1044.

Evans JD (2006) Beepath: An ordered quantitative-PCR array for exploring honey bee immunity and disease. J Invertebr Pathol 93:135-139.

Feng M, Fang Y and Li J (2009) Proteomic analysis of honeybee worker (Apis mellifera) hypopharyngeal gland development. BMC Genomics 10:645.

Ferrari S, Sanguinetti M, De Bernardis F, Torelli R, Posteraro B, Vandeputte P and Sanglard D (2011) Loss of mitochondrial functions associated with azole resistance in Candida glabrata results in enhanced virulence in mice. Antimicrob Agents Chemother 55:1852-1860.

Gerhold D, Meiqing LU, Jian XU, Austin C, Caskey CT and Rushmore T (2001) Monitoring expression of genes involved in drug metabolism and toxicology using DNA microarrays. Physiol Genomics 5:161-170.

Gilbert DG (2007) DroSpeGe: Rapid access database for new Drosophila species genomes. Nucleic Acids Res 35:D480D485.
Grozinger CM, Sharabash NM, Whitfield CW and Robinson GE (2003) Pheromone -mediated gene expression in the honey bee brain. Proc Natl Acad Sci U S A 100:14519-14525.

Hyink O, Laas F and Dearden PK (2013) Genetic tests for alleles of complementary -sex-determiner to support honeybee breeding programmes. Apidologie 44:306-313.

Ji T, Liu Z, Shen J, Shen F, Liang Q, Wu L, Chen G and Corona M (2014) Proteomics analysis reveals protein expression differences for hypopharyngeal gland activity in the honeybee, Apis mellifera carnica Pollmann. BMC Genomics 15:665.

Jianke L, Mao F, Begna D, Yu F and Aijuan Z (2010) Proteome comparison of hypopharyngeal gland development between Italian and royal jelly producing worker honeybees (Apis mellifera L.). J Proteome Res 9:6578-6594.

Klein A-M, Vaissiere BE, Cane JH, Steffan-Dewenter I, Cunningham SA, Kremen C and Tscharntke T (2007) Importance of pollinators in changing landscapes for world crops. Proc R Soc Lond B Biol Sci 274:303-313.

Kucharski R and Maleszka R (2002) Evaluation of differential gene expression during behavioral development in the honeybee using microarrays and northern blots. Genome Biol 3:1-7.

Li J, Li H and Zhang L (2008) Analysis of the proteome of the larvae of the high royal jelly producing worker bees (Apis mellifera L.) Sci Agric Sinica 41:880-889.

Li WF, Huang ZY, Liu F, Li ZG, Yan LM, Zhang SW, Chen SL, Zhong BX and Su SK (2013) Molecular cloning and characterization of juvenile hormone acid methyltransferase in the honey bee, Apis mellifera, and its differential expression during caste differentiation. PloS One 8:e68544.

Liu F, Li W, Li Z, Zhang S, Chen S and Su S (2011) Highabundance mRNAs in Apis mellifera: Comparison between nurses and foragers. J Insect Physiol 57:274-279.

Livak KJ and Schmittgen TD (2001) Analysis of relative gene expression data using real-time quantitative PCR and the $2^{-\triangle \Delta C T}$ method. Methods 25:402-408.

Mao F, Yu F and Li J (2009) Proteomic analysis of honeybee worker (Apis mellifera) hypopharyngeal gland development. BMC Genomics 10:645.

Martins JR, Nunes FMF, Simões ZLP and Bitondi MMG (2008) A honeybee storage protein gene, hex $70 a$, expressed in developing gonads and nutritionally regulated in adult fat body. J Insect Physiol 54:867-877.

Martins JR, Nunes FM, Cristino AS, Simões ZL and Bitondi MM (2010) The four hexamerin genes in the honey bee: Structure, molecular evolution and function deduced from expression patterns in queens, workers and drones. BMC Mol Biol 11:23.

Menuz K, Larter NK, Park J and Carlson JR (2014) An RNA-seq screen of the Drosophila antenna identifies a transporter necessary for ammonia detection. PLoS Genet 10:e1004810

Morse RA and Calderone NW (2000) The value of honey bees as pollinators of US crops in 2000. Bee Culture 128:1-15.

Navajas M, Migeon A, Alaux C, Martin-Magniette M-L, Robinson G, Evans J, Cros-Arteil S, Crauser D and Conte Y (2008) Differential gene expression of the honey bee Apis mellifera associated with Varroa destructor infection. BMC Genomics 9:301.

Nie H, Liu C, Cheng T, Li Q, Wu Y, Zhou M, Zhang Y and Xia Q (2014) Transcriptome analysis of integument differentially 
expressed genes in the pigment mutant (quail) during molting of silkworm, Bombyx mori. PloS One 9:e94185.

Nomura S, Takahashi J-I, Sasaki T, Yoshida T and Sasaki M (2009) Expression of the dopamine transporter in the brain of the honeybee, Apis mellifera L.(Hymenoptera: Apidae). Appl Entomol Zool 44:403-411.

Ohashi K, Natori S and Kubo T (1997) Change in the mode of gene expression of the hypopharyngeal gland cells with an age-dependent role change of the worker honeybee Apis mellifera L. Eur J Biochem 249:797-802.

Page Jr RE, Rueppell O and Amdam GV (2012) Genetics of reproduction and regulation of honey bee (Apis mellifera L.) social behavior. Annu Rev Genet 46:97-119.

Perk CG and Mercer AR (2006) Dopamine modulation of honey bee (Apis mellifera) antennal -lobe neurons. J Neurophysiol 95:1147-1157.

Ramadan MF and Al-Ghamdi A (2012) Bioactive compounds and health-promoting properties of royal jelly: A review. J Funct Foods 4:39-52.

Scharlaken B, de Graaf DC, Goossens K, Brunain M, Peelman LJ and Jacobs FJ (2008) Reference gene selection for insect expression studies using quantitative real-time PCR: The head of the honeybee, Apis mellifera, after a bacterial challenge. $\mathrm{J}$ Insect Sci 8:33.

Schmidt JO (1997) Bee products. In: Mizrahi A and Lensky Y (eds) Bee Products. Springer, New York, pp 15-26.

Schmitzová J, Klaudiny J, Albert S, Schröder W, Schreckengost W, Hanes J, Júdová J and Simúth J (1998) A family of major royal jelly proteins of the honeybee Apis mellifera L. Cell Mol Life Sci 54:1020-1030.

Schultz W (1992) Activity of dopamine neurons in the behaving primate. Semin Neurosci 4:129-138.

Su S and Chen S (2003) Research on morphological genetic marker of honeybee (Apis mellifera ligustica) in royal jelly production performance. Hereditas 25:677-680.

Tang QY and Zhang CX (2013) Data Processing System (DPS) software with experimental design, statistical analysis and data mining developed for use in entomological research. Insect Sci 20:254-260.

Telfer WH and Kunkel JG (1991) The function and evolution of insect storage hexamers. Annu Rev Entomol 36:205-228.

The Honey Bee Genome Sequencing Consortium (2006) Insights into social insects from the genome of the honeybee Apis mellifera. Nature 443:931-949.
Toth AL, Kantarovich S, Meisel AF and Robinson GE (2005) Nutritional status influences socially regulated foraging ontogeny in honey bees. J Exp Biol 208:4641-4649.

Vergoz V, Roussel E, Sandoz JC and Giurfa M (2007) Aversive learning in honeybees revealed by the olfactory conditioning of the sting extension reflex. PLoS One 2:e288.

Viuda-Martos M, Ruiz-Navajas Y, Fernández-López J and Pérez-Álvarez JA (2008) Functional properties of honey, propolis, and royal jelly. J Food Sci 73:R117-R124.

Whitfield CW, Cziko A-M and Robinson GE (2003) Gene expression profiles in the brain predict behavior in individual honey bees. Science 302:296-299.

Wise RA (2004) Dopamine, learning and motivation. Nat Rev Neurosci 5:483-494.

Zahurak M, Parmigiani G, Yu W, Scharpf RB, Berman D, Schaeffer E, Shabbeer S and Cope L (2007) Pre-processing Agilent microarray data. BMC Bioinformatics 8:142.

Zhang L, Li J-K and Wu L-M (2007) Profile analysis of the proteome of the egg of the high royal jelly producing bees (Apis mellifera L.). Agric Sci China 6:1138-1148.

Zhang S, Schwarz S, Pahl M, Zhu H and Tautz J (2006) Honeybee memory: A honeybee knows what to do and when. J Exp Biol 209:4420-4428.

Zheng A, Fang Y, Feng M, Wu J and Song F, Li J (2010) Proteome comparison between worker pupal head of native Italian honeybee (Apis mellifera L.) and higher royal jelly producing strain. Sci Agric Sinica 43:1703-1715.

\section{Supplementary material}

The following online information is available for this article:

Table S1 - The details of probes in microarray construction. Table S2 - Standardized data of high and low royal jelly bees.

Table S3 - Primer sequences of the detected genes.

Table S4 - Information of 369 differentially expressed genes screened by gene chip.

Table S5 - KEGG analysis of the differently expressed genes.

Associate Editor: Ana Tereza R. Vasconcelos

License information: This is an open-access article distributed under the terms of the Creative Commons Attribution License (type CC-BY), which permits unrestricted use, distribution and reproduction in any medium, provided the original article is properly cited. 